\title{
The Obscured Bilateral Testicular Tumors: Is It Adrenal Rest Tumors or Leydig Cell Tumors
}

\author{
${ }^{1 *}$ Mohammed S. Al-Marhoon, ${ }^{2}$ Intisar Al-Hashmi, ${ }^{3}$ Khalid Rehman \\ ${ }^{1,2,3}$ Urology Division, Department of Surgery, College of Medicine \& Health Sciences, \\ Sultan Qaboos University, Oman \\ *msalmarhoon@gmail.com,mmarhoon@squ.edu.om \\ ${ }^{4}$ Omayma Elshafi, ${ }^{5}$ Anna Saparamadu \\ ${ }^{4,5}$ Department of Medicine, College of Medicine \& Health Sciences \\ Sultan Qaboos University, Oman
}

\begin{abstract}
A young male presented with infertility and found to have bilateral testicular masses with obscured past history of Congenital Adrenal Hyperplasia (CAH) since childhood. The differential diagnosis of bilateral testicular masses includes: Adrenal Rest tumors, Leydig cell tumors and germ cell tumors. A battery of biochemical investigations in addition to surgical exploration are needed to reach to the final diagnosis. However, differentiating Leydig cell tumors from the tumors of the adrenogenital syndrome can be difficult and need an alert urologist with collaboration of pathologist and endocrinologist.
\end{abstract}

Keywords: Bilateral, Testicular tumors, Adrenal rest tumors, Leydig cell tumors

Abbreviations: GCTs: germ cell tumors; ITGCN: intratubular germ cell neoplasia

\section{INTRODUCTION}

Germ cell tumors (GCTs) are relatively rare malignancies, accounting for 1-2\% of cancers among men in the United States, with an incidence of approximately 5 cases per 100,000. Approximately $90 \%$ of GCTs arise in the testis and $2-5 \%$ are extragonadal (the retroperitoneum and mediastinum are the most common sites). ${ }^{1}$ The incidence of bilateral GCTs is approximately $2 \% .^{2}$ Testicular lymphoma is less common than GCTs but accounts for the majority of testicular tumors in men older than 50 years of age and is more likely to have a synchronous bilateral presentation. There are four well-established risk factors for testicular cancer: cryptorchidism, family history of testicular cancer, a personal history of testicular cancer, and intratubular germ cell neoplasia (ITGCN). Infertile men also have a higher incidence of testicular cancer. Sex cord/stromal tumors are rare, comprising approximately $4 \%$ of testicular neoplasms, and the term refers to neoplasms containing Leydig cells, Sertoli cells, granulosa cells, or thecal cells. ${ }^{1}$ This case report highlights the dilemma of differential diagnosis of bilateral testicular masses.

\section{Case Report}

A 28 -years old male presented to a private infertility clinic for the evaluation of his infertility. He is married for 4 years with no children. He was found to have bilateral scrotal masses and hence referred to our hospital for further evaluation. On further history he is known to have Bilginturan syndrome (Brachydactyly- short stature - hypertension), Congenital Adrenal hyperplasia (11-hydroxylase deficiency) with uncontrolled hypertension and noncompliant to medications and lost to follow up for 10 years, primary infertility with azospermia and picture of hypogonadotropic hypogonadism. He had bilateral varicocelectomy and testicular biopsy elsewhere in 2011 and the histopathology report showed no sperms or malignancy. On examination he had short stature and small hands with normal secondary sexual features and no gynecomastia or thyroid swellings. His blood pressure was 112/60 mmgh (on antihypertenstion medication). His abdominal examination was unremarkable. There was a hard irregular right testicular mass measuring $3 \times 3 \mathrm{~cm}$ in size with a lax hydrocele. The left testis also had a hard irregular testicular mass measuring $4 \times 5 \mathrm{~cm}$ in size with a lax hydrocele (Figure 1). On digital rectal examination the prostate was small and smooth. The working diagnosis was primary infertility with bilateral testicular masses to rule out testicular cancer. Based on this and his past history the differential diagnosis of the testicular masses included: Adrenal Rest tumors, Lydige cell 
tumor and germ cell tumors. The following investigations were done: complete blood count (normal), coagulation (normal), renal function test (normal), thyroid function tests (normal), liver function tests (normal), blood glucose, tumor markers (Alpha Fetoprotein 2 KIU/L, HCG <0.5 IU/L, LDH 221 U/L) were normal; The hormones were as follows: FSH <0.2 IU/L (Low), LH 0.3 IU/L (low), Prolactin 234 mIU/L (normal), Cortisol 278 nmol/L (normal), 11-Deoxycortisol 314.9 ng/ml (high), ACTH 81.5 pmol/L (high), Testosterone $40.7 \mathrm{nmol} / \mathrm{L}$ (high), DHEAS 10.9 umol/L (normal), Estradiol $0.54 \mathrm{nmol} / \mathrm{L}$ (high). HIV, hepatitis and syphilis were all negative. Karyotype, urine culture, chest xray and ultrasound of testis and CT abdomen were done. CT abdomen showed bilateral massive hyperplasia of both adrenal glands with preserved adeniform contour and presence of multiple nodules bilaterally- largest measuring $1.9 \mathrm{~cm}$ on right side and $3.5 \mathrm{~cm}$ on left side (Figure 2).

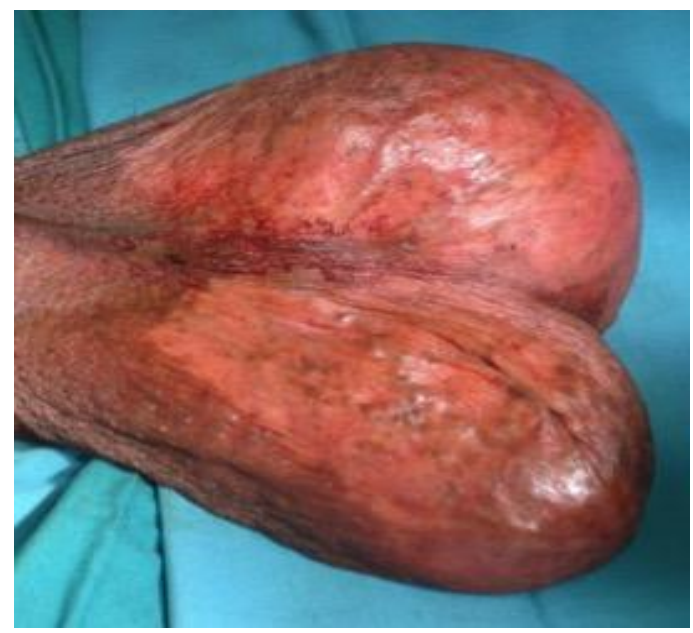

Figure1. Clinical picture of bilateral testicular masses

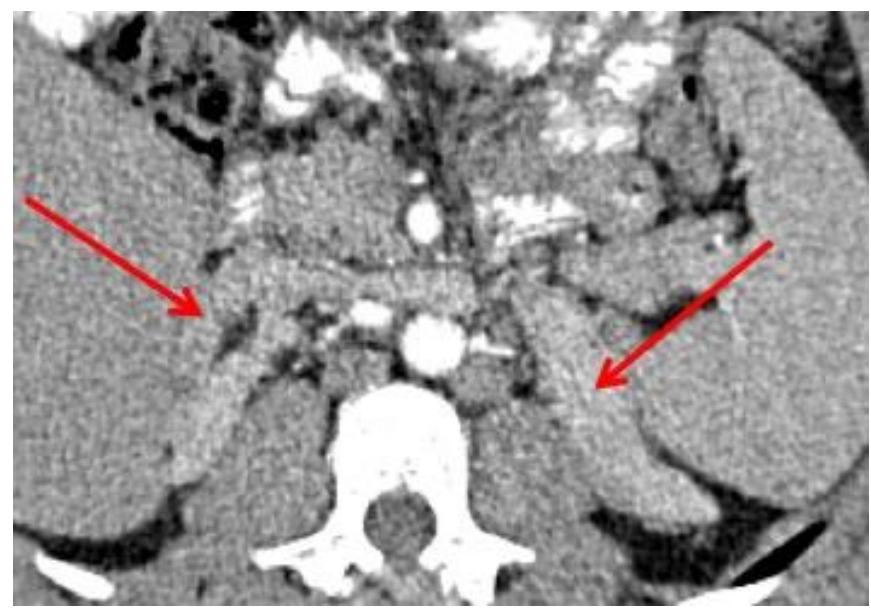

Figure2. CT of abdomen showing bilateral adrenal enlargement

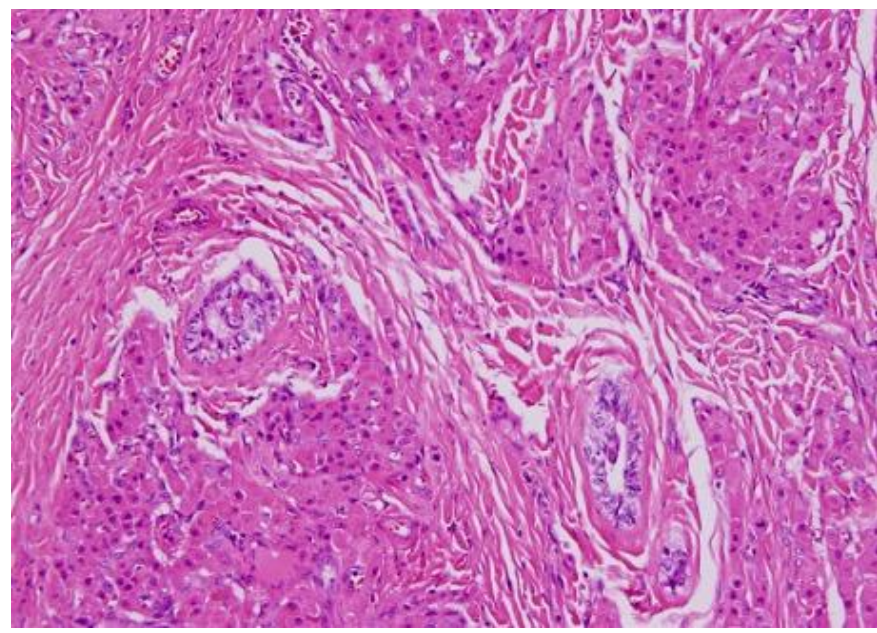

Figure3. Histopathology of the right orchiectomy with morphology consistent with Leydig cell tumor 
The patient was taken for bilateral inguinal exploration including frozen section, sperm banking, testis-conservative surgery when applicable. Right orchiectomy was done. The histopathology reported was as follows: Right orchiectomy and frozen sections of tumors (from both slides), H\&E and IHC morphology consistent with bilateral Leydig cell tumor (Figure 3). No evidence of malignancy. Testicular atrophy is present with no evidence of intratubular germ cell neoplasia. On Follow up the patient was advised to have bilateral laparoscopic adrenalectomy for the control of hypertention and to continue steroid replacement for life.

\section{DISCUSSION}

Leydig cell tumors are the most common of the sex cord/stromal tumors. There is no association with cryptorchidism. Most of these tumors occur in adult males between age 20 and 60 years, although approximately one fourth occur in children. In adults, the most common presentation is a painless testicular mass, frequently with feminizing characteristics (as a result of androgen excess and peripheral conversion to estrogen), including gynecomastia, impotence, and decreased libido and infertility. ${ }^{3}$ Children usually present with a testicular mass and pseudopuberty (prominent external genitalia, pubic hair growth, and masculine voice) ${ }^{4}$ Ninety percent of these tumors are benign, and $10 \%$ are malignant. ${ }^{5}$ Advanced patient age, larger tumor size, presence of vascular and margin section infiltration are signs of negative prognosis. The presence of metastatic disease is the only reliable criteria for making the distinction between benign and malignant tumors. The most frequent metastatic sites are the retroperitoneum and lung. ${ }^{4}$ Radical inguinal orchiectomy is the initial treatment of choice for Leydig cell tumor, with $85 \%$ diagnosed on frozen section intraoperatively. ${ }^{6}$ However, when diagnosed and treated early, testicle-sparing surgery have been proved to be a feasible and safe choice. ${ }^{7}$ RPLND is reasonable in select cases with adverse features. Surveillance of men with Leydig cell tumors is not necessary in those without clinical or pathologic features suggestive of malignancy. Metastatic Leydig cell tumors are resistant to chemotherapy and radiation therapy.

Congenital adrenal hyperplasia $(\mathrm{CAH})$ is an inherited disorder of adrenal steroid synthesis. In more than $90 \%$ of cases it is caused by 21-hydroxylase deficiency leading to glucocorticoid deficiency mineralocorticoid deficiency, and in less than $5 \%$ of cases it is caused by 11 -hydroxylase deficiency leading to increased levels of deoxycorticosterone that causes hypertension and hypokalaemia. ${ }^{8}$ The compensatory increase in adrenocorticotropic hormone (ACTH) secretion by the pituitary gland leads to stimulation of the adrenals and consequently overproduction of the adrenal androgen precursors dehydroepiandrosterone and androstenedione and subsequently of active androgens. ${ }^{9}$ One of the most serious problems in adult CAH patients is infertility. ${ }^{10}$ The most important cause of male infertility in $\mathrm{CAH}$ patients is the presence of testicular tumours resulting in primary gonadal failure. Because of the morphological and functional resemblance to adrenal tissue, they are called testicular adrenal rest tumours (TART). They are always benign, although compression of the seminiferous tubules may lead to obstructive azoospermia, irreversible damage of the surrounding testicular tissue, and consequently infertility. ${ }^{11-13}$

Differentiating Leydig cell tumors from the tumors of the adrenogenital syndrome can be difficult, and testis tumors associated with adrenogenital syndrome presenting at later age are frequently misdiagnosed as Leydig cell tumors. ${ }^{14}$ Clinical differences can assist in the diagnosis. Only $3 \%$ of Leydig cell tumors are bilateral. In contrast, $80 \%$ of testis tumors associated with the adrenogenital syndrome involve both gonads. ${ }^{4}$ Malignancy has not been reported in cases of testis tumors of adrenogenital syndrome but is reported in $10 \%$ of Leydig cell tumors in adults. Children with Leydig cell tumors have isosexual precocity as a consequence of testosterone production by the tumor. In addition, it is not uncommon for previously reported cases of bilateral Leydig cell tumors to be reclassified as tumors of the adrenogenital syndrome after additional histologic and biochemical analysis. ${ }^{8}$ Because of their considerable pathologic similarity, a complete biochemical and histopathologic evaluation is required to differentiate the two entities. ${ }^{15}$ When the biochemical profile is diverse and steroid levels do not clearly define the tumor of origin, dexamethasone suppression and adrenocorticotropic hormone (ACTH) stimulation tests may help in making the correct diagnosis. Leydig cell tumors are autonomous and should not respond to either suppression or stimulation tests, whereas tumors of the adrenogenital syndrome do. ${ }^{15}$

\section{Conclusions}

A young male presented with infertility and found to have bilateral testicular masses with obscured past history of Congenital Adrenal Hyperplasia (CAH) since childhood with poor compliance for 
medications has put the treating urologist in a delima of the origin of the testicular masses. The differential diagnosis of the testicular masses included: Adrenal Rest tumors, Lydige cell tumor and germ cell tumors. A battery of biochemical investigations in addition to surgical exploration are needed to reach to the final diagnosis. However, differentiating Leydig cell tumors from the tumors of the adrenogenital syndrome can be difficult and need an alert urologist with collaboration of pathologist and endocrinologist.

\section{REFERENCES}

[1] Masur Y, Steffens J, Ziegler M, et al. Leydig cell tumors of the testis--clinical and morphologic aspects. Urologe A 1996; 35: 468-71.

[2] Fosså SD, Chen J, Schonfeld SJ, et al. Risk of contralateral testicular cancer: A population-based study of 29515 U.S. Men. J Natl Cancer Inst 2005; 97: 1056-1066.

[3] Fallick ML, Lin WW, Lipshultz LI. Leydig cell tumors presenting as azoospermia. J Urol 1999; 161: 1571-2.

[4] Kim I, Young RH, Scully RE. Leydig cell tumors of the testis. A clinicopathological analysis of 40 cases and review of the literature. Am J Surg Pathol 1985; 9: 177-92.

[5] Grem JL, Robins HI, Wilson KS, et al. Metastatic Leydig cell tumor of the testis. Report of three cases and review of the literature. Cancer 1986; 58: 2116-9.

[6] Carmignani L, Salvioni R, Gadda F, et al. Long-term followup and clinical characteristics of testicular Leydig cell tumor: experience with 24 cases. J Urol 2006; 176: 2040-3; discussion 2043.

[7] Bozzini G, Picozzi S, Gadda F, et al. Long-term follow-up using testicle-sparing surgery for Leydig cell tumor. Clin Genitourin Cancer 2013; 11: 321-4.

[8] Rich MA, Keating MA, Levin HS, et al. Tumors of the adrenogenital syndrome: an aggressive conservative approach. J Urol 1998; 160: 1838-41.

[9] Speiser PW, White PC. Congenital adrenal hyperplasia. N Engl J Med 2003; 349: 776-88.

[10] Cabrera MS, Vogiatzi MG, New MI. Long term outcome in adult males with classic congenital adrenal hyperplasia. J Clin Endocrinol Metab 2001; 86: 3070-8.

[11] Chihaoui M, Kanoun F, Chaker F, et al. Testicular adrenal rest tumours in young adult males with congenital adrenal hyperplasia: prevalence and impact on testicular function. Andrologia 2015.

[12] Claahsen-van der Grinten HL, Hermus ARMM, Otten BJ. Testicular adrenal rest tumours in congenital adrenal hyperplasia. Int J Pediatr Endocrinol 2009; 2009: 624823.

[13] Rajkanna J, Oyibo SO. Large testicular adrenal rest tumours in a patient with congenital adrenal hyperplasia. Endocrinol diabetes Metab case reports 2015; 2015: 140080.

[14] Rutgers JL, Young RH, Scully RE. The testicular 'tumor' of the adrenogenital syndrome. A report of six cases and review of the literature on testicular masses in patients with adrenocortical disorders. Am J Surg Pathol 1988; 12: 503-13.

[15] Rich MA, Keating MA. Leydig cell tumors and tumors associated with congenital adrenal hyperplasia. Urol Clin North Am 2000; 27: 519-28. 